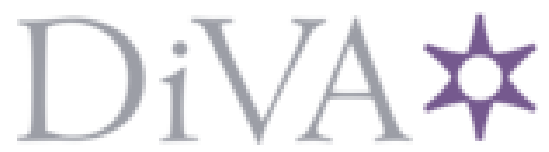

http://www.diva-portal.org

\title{
Postprint
}

This is the accepted version of a paper published in Methods in Molecular Biology. This paper has been peer-reviewed but does not include the final publisher proof-corrections or journal pagination.

Citation for the original published paper (version of record):

Tengholm, A., Idevall-Hagren, O. (2015)

Imaging sub-plasma membrane cAMP dynamics with fluorescent translocation reporters.

Methods in Molecular Biology, 1294: 85-101

http://dx.doi.org/10.1007/978-1-4939-2537-7_7

Access to the published version may require subscription.

N.B. When citing this work, cite the original published paper.

Permanent link to this version:

http://urn.kb.se/resolve?urn=urn:nbn:se:uu:diva-300477 


\section{Imaging sub-plasma membrane cAMP dynamics with fluorescent translocation reporters}

Anders Tengholm* and Olof Idevall-Hagren

Department of Medical Cell Biology, Uppsala University, Biomedical Centre, Box 571, SE-75123 Uppsala, Sweden

Running head: Imaging sub-membrane cAMP dynamics

Keywords: cAMP oscillations, plasma membrane, protein kinase A, translocation, total internal reflection fluorescence

*Correspondence:

Prof. Anders Tengholm

E-mail: anders.tengholm@mcb.uu.se

Phone: +46-18-4714481 


\section{Summary}

Imaging cAMP dynamics in single cells and tissues can provide important insights into the regulation of a variety of cellular processes. In recent years a large number of tools for cAMP measurements have been developed. While most cAMP reporters are designed to undergo changes in fluorescence resonance energy transfer (FRET) there are alternative techniques with advantages for certain applications. Here we describe protocols for cAMP measurements in the sub-plasma membrane space based on detection of the cAMP-induced translocation of engineered fluorescent-protein-tagged subunits of protein kinase A between the cytoplasm and the plasma membrane. Total internal reflection fluorescence (TIRF) imaging of the changes in reporter localization yields robust signal changes and has contributed to the discovery of cAMP oscillations in the sub-plasma membrane space of insulin-secreting $\beta$-cells stimulated with glucose and gluco-incretin hormones. We also demonstrate how the technique can be combined with measurements of the cytosolic $\mathrm{Ca}^{2+}$ concentration or with recordings of the sub-cellular localization of the cAMP effector protein Epac2. The translocation reporter approach provides a valuable complement to other methods for imaging sub-membrane cAMP dynamics in various types of cells.

\section{Introduction}

Second messenger signaling is highly dynamic and often involves distinct temporal patterns and spatial compartmentalization, which help to achieve efficiency and specificity in the control of downstream cellular functions. Such spatio-temporal dynamics have been particularly well recognized for $\mathrm{Ca}^{2+}$, thanks to the early advent of fluorescent indicators to measure the cytoplasmic $\mathrm{Ca}^{2+}$ concentration in individual cells (1). Measurements of intracellular cAMP dynamics have been more challenging 
and were for a long time restricted by a lack of suitable tools. There are now a number of available methods for real-time measurements of cAMP (reviewed in $(2,3)$ and described elsewhere in this volume). Most cAMP indicators are based on fluorescence resonance energy transfer (FRET). The first version required cumbersome microinjections of fluorescently labeled regulatory and catalytic subunits of protein kinase A (PKA) (4) and suffered from distorted signal as the probe moieties gradually became paired with endogenous subunits during repeated cycles of activation. These problems were overcome by genetically encoded versions of the sensor $(5,6)$. FRET probes with the additional advantage of being expressed as a single polypeptide chain were soon generated from the other cAMP target proteins Epac1 and Epac2 as well as from isolated cAMP-binding domains from PKA, Epac and the hyperpolarization activated cyclic nucleotide-gated potassium channel 2 (7-10).

Detection of FRET is not always trivial, however, and signal changes are often rather small and the readout prone to various artifacts $(2,11,12)$. Alternative classes of cAMP sensor include cyclic nucelotide-gated (CNG) channels (13), the activity of which are monitored with patch-clamp current recordings or as changes of the intracellular $\mathrm{Ca}^{2+}$ concentration. Such channels have the advantage of responding quickly and of reporting cAMP in the sub-plasma membrane space where the adenylate cyclases are located and where many important signaling and physiological events take place. Drawbacks with the CNG channel sensors are that their expression may affect the electrophysiological activity of the cell and that the $\mathrm{Ca}^{2+}$ entry may modulate adenylate cyclase and phosphodiesterase activities, thereby affecting the cAMP signal that is being measured.

Another method, particularly useful for recording sub-plasma membrane cAMP dynamics, is based on protein translocation as a readout (14). Like the early versions 
of the FRET biosensors $(4,5)$, it takes advantage of the fact that the regulatory and catalytic subunits of PKA dissociate following binding of cAMP to the regulatory subunit. By targeting the regulatory subunit to the plasma membrane, the holoenzyme becomes located at the membrane under basal conditions (14). As the sub-membrane cAMP concentration increases the holoenzyme dissociates and the change in localization of the catalytic subunit can be monitored with fluorescence microscopy simply as a change in localization. This translocation causes a signal change that is much larger than the simultaneously occurring loss of FRET.

We have generated such a translocation sensor with a modified PKA RII $\beta$ regulatory subunit, similar to that in the original genetically encoded FRET-based sensor. The regulatory subunits of PKA dimerize and in addition, subunits of the RII type are often targeted to A-kinase anchoring proteins (AKAPs) by interactions with their $\mathrm{N}$ terminal region (15). To avoid mistargeting of the sensor, ninety amino acid residues, including both the AKAP-binding and the dimerization regions, were deleted. Cyan fluorescent protein (CFP) was added to the C-terminus (see Note 1) and the fluorescent protein was in turn extended by a C-terminal polybasic stretch and a CAAX-motif from K-Ras (see Note 2). The CAAX-motif becomes posttranslationally farnesylated and this lipid modification together with the basic amino acid residues targets the protein to the plasma membrane (Fig 1). The PKA catalytic subunit $\mathrm{C} \alpha$ was used in its full length wildtype form and tagged with a yellow fluorescent protein (YFP) at its C-terminus (see Note 3).

The localization of the reporter can be visualized with confocal microscopy, with which the translocation of the fluorescent $\mathrm{C} \alpha$ subunit can be detected either as changes in membrane or cytoplasmic fluorescence (Fig 2). However, a better approach is to use total internal reflection fluorescence (TIRF) microscopy, which 
selectively illuminates a volume within $<100 \mathrm{~nm}$ of the plasma membrane that adheres to the coverslip (16). The translocation of the catalytic subunit will then be observed as changes in fluorescence intensity in the cell footprint and can easily be detected without complicated image analysis to define sub-cellular structures (Fig $3 \mathrm{~A})$. The regulatory subunit of the reporter is constitutively present in the membrane and can therefore be used as a reference to obtain a ratiometric readout of the signal. If there is no need for high spatial resolution, translocation can be recorded from many cells at the same time using a low magnification TIRF imaging setup (Fig 3B). The translocation reporter for cAMP has been used to detect sub-membrane cAMP signals in insulin-secreting $\beta$-cells. For example, the technique allowed the demonstration that activation of the receptors for glucagon and glucagon-like peptide1 (GLP-1), which are linked via Gas to adenylate cyclases, not only evoked a rise of cAMP but that there were pronounced oscillations of the sub-membrane cAMP concentration (14)(Fig 4). These dynamic changes were not unique to receptor stimuli and it was also discovered that glucose, the major stimulator of insulin secretion from $\beta$-cells, evoked cAMP oscillations (17). Glucose exerts its action after uptake and metabolism in the $\beta$-cell. The mechanisms underlying the glucose-induced cAMP generation are not clear, but it seems that both the stimulated formation of ATP by cell metabolism and the resulting increase of the cytoplasmic $\mathrm{Ca}^{2+}$ concentration is important for the cAMP response $(17,18)$ (Fig 3B). It is likewise unclear what feedback loops participate in the generation of oscillations, but they may involve changes in the sub-membrane concentrations of ATP, $\mathrm{Ca}^{2+}$ and activities of phosphodiesterases $(\mathbf{1 9}, 20)$.

The translocation reporter for cAMP has not only been employed for sub-membrane cAMP recordings in various types of dispersed cells, including, apart from $\beta$-cells, 
breast cancer cells (21) and embryonic stem cell-derived neurons (22), but also for detection of cAMP changes in cells within the intact pancreatic islet micro-organ (18). The reporter has also been used in combination with measurements of other intracellular messengers, including $\mathrm{Ca}^{2+}$ and phosphatidylinositol-3,4,5-trisphosphate $\left[\operatorname{PtdIns}(3,4,5) P_{3}\right](23)$, as well as the membrane binding of other proteins, including the cAMP effector protein Epac2 (24). The present protocol describes measurements of the sub-membrane cAMP dynamics in clonal MIN6 $\beta$-cells (25) and intact islets of Langerhans using translocation-based reporters as well as how cAMP can be recorded together with the cytoplasmic $\mathrm{Ca}^{2+}$ concentration and the sub-cellular localization of the Epac2 protein (Fig 4).

\section{Materials}

\subsection{Cell culture and transfection}

1. Glass cover slips, 25-mm diameter, 0.13-0.19 mm thickness (from e.g. MenzelGläser, Germany).

2. Poly-L-lysine (MW 70,000-150,000). Stock solution: $0.1 \mathrm{mg} / \mathrm{mL}$ poly-L-lysine in sterile water, stored at $-20{ }^{\circ} \mathrm{C}$. Working solution: $0.01 \mathrm{mg} / \mathrm{mL}$ poly-L-lysine in sterile water.

3. Dulbecco's phosphate buffered saline (D-PBS):137 mM NaCl, $2.67 \mathrm{mM} \mathrm{KCl}, 8.10$ $\mathrm{mM} \mathrm{Na}_{2} \mathrm{HPO}_{4}, 1.47 \mathrm{mM} \mathrm{KH}_{2} \mathrm{PO}_{4}$, store at $+4{ }^{\circ} \mathrm{C}$. 
4. Complete growth medium for MIN6 $\beta$-cells: Dulbecco's Modified Eagle's Medium (DMEM) containing $4500 \mathrm{mg} / \mathrm{L}$ glucose and supplemented with $15 \%(\mathrm{v} / \mathrm{v})$ fetal calf serum, $2 \mathrm{mM}$ L-glutamine, $70 \mu \mathrm{M} \beta$-mercaptoethanol, $100 \mathrm{U} / \mathrm{mL}$ penicillin and 100 $\mu \mathrm{g} / \mathrm{mL}$ streptomycin. Store at $+4{ }^{\circ} \mathrm{C}$.

5. Lipofectamine ${ }^{\mathrm{TM}} 2000$ reagent (Life Technologies, Invitrogen).

6. OptiMEM I (Life Technologies, Invitrogen).

7. Plasmid DNA encoding the two fluorescent reporter moieties $\triangle$ RII $\beta$-CFP-CAAX and C $\alpha$-YFP. Multiparameter experiments may require vectors encoding additional fluorescence-tagged proteins of interest.

8. Fura Red acetoxymethyl ester (Life Technologies, Molecular Probes).

\subsection{Fluorescence microscopy}

1. Experimental buffer: $125 \mathrm{mM} \mathrm{NaCl}, 4.9 \mathrm{mM} \mathrm{KCl}, 1.3 \mathrm{mM} \mathrm{CaCl}_{2}, 1.2 \mathrm{mM} \mathrm{MgCl}_{2}$, $25 \mathrm{mM}$ HEPES, $3 \mathrm{mM}$ D-glucose and $0.1 \%(\mathrm{w} / \mathrm{v})$ bovine serum albumin. Adjust $\mathrm{pH}$ to 7.40 at $37^{\circ} \mathrm{C}$ with $\mathrm{NaOH}$ (see Note 4 ).

2. Confocal or TIRF fluorescence microscopes: Visualization of probe translocation between the cytoplasm and plasma membrane requires a microscope with optical sectioning capability. Confocal imaging works well, but TIRF has advantages for monitoring changes of plasma membrane fluorescence since it does not require detailed sub-cellular image analysis (see Note 5). 
3. Light source, filters for wavelength selection, and equipment for image acquisition and analysis (see Note 6):

a. Lasers appropriate for exciting fluorescent proteins (see (Note 7).

b. Appropriate excitation filters (e.g. $442 \mathrm{~nm} / 10 \mathrm{~nm}$ half-bandwidth and 514/10 nm for CFP and YFP), dichroic mirror (e.g. ZT442/514rpc) and emission filters (e.g. 483/32 $\mathrm{nm}$ and 542/27 $\mathrm{nm}$ for CFP and YFP) (see Note 8)

c. A sensitive low-light level charge-coupled device (CCD) or complementary metaloxide semiconductor (CMOS) camera for spinning disk confocal or TIRF microscope can be obtained from e.g. Andor Technology, Hamamatsu, and Roper Scientific.

d. Data acquisition and analysis software (see Note 9)

4. Equipment for buffer superfusion and thermoregulation (see Note 10).

\section{Methods}

\subsection{Preparation of poly-L-lysine coated coverslips}

1. Wash the coverslips in deionized water and sterilize in a heat incubator $\left(180^{\circ} \mathrm{C}\right)$ or by dipping them into $95 \%$ ethanol and passing through the flame of a Bunsen burner. Place each sterile coverslip in a $35 \mathrm{~mm}$ diameter culture dish.

2. Place $\mathrm{a} \sim 100 \mu \mathrm{L}$ drop of poly-L-lysine $(0.01 \mathrm{mg} / \mathrm{mL})$ in the centre of the coverslip, leave it for 20-30 min at room temperature and then remove the poly-L-lysine and 
rinse the coverslip twice with sterile water. It is important not to let the poly-L-lysine dry on the coverslips. After the last washing step, remove as much water as possible and allow the coverslips to dry in a laminar flow bench with the lids of the culture dishes open (see Note 11).

\subsection{Cell culture and transient transfection}

1. Seed cells onto the dried poly-L-lysine coated coverslips by adding $3 \mathrm{~mL}$ cell suspension at a concentration of $0.3-0.6 \times 10^{6}$ cells $/ \mathrm{mL}$. The confluency should be approximately $50 \%$ on the day of transfection (see Note 12).

2. For each coverslip to be transfected, mix $1 \mu \mathrm{g}$ of plasmid DNA encoding each component of the cAMP translocation biosensor with $125 \mu \mathrm{L}$ OptiMEM I in one tube and $5 \mu \mathrm{L}$ Lipofectamine $^{\mathrm{TM}} 2000$ with $125 \mu \mathrm{L}$ OptiMEM I in another tube. Leave the tubes for $5 \mathrm{~min}$ at room temperature (see Note 13).

3. Combine the content of the two tubes and mix gently (do not vortex). Leave for 20 min at room temperature to allow DNA-liposome complexes to form.

4. Remove complete culture medium from the cells and carefully rinse them once with D-PBS preheated to $37^{\circ} \mathrm{C}$.

5. Remove the D-PBS and add to each coverslip $750 \mu \mathrm{L}$ OptiMEM I conditioned to $37^{\circ} \mathrm{C}$. Subsequently add the DNA-liposome combination $(\sim 250 \mu \mathrm{L}$ per coverslip) and mix by gently rocking the culture dish back and forth. 
6. Incubate the cells for $3-5 \mathrm{~h}$ at $37{ }^{\circ} \mathrm{C}$ and $5 \% \mathrm{CO}_{2}$ in a cell culture incubator.

7. Terminate the transfection reaction by removing the transfection medium, rinsing the cells once with D-PBS at $37^{\circ} \mathrm{C}$ and adding $3 \mathrm{~mL}$ complete growth medium.

8. Culture the transfected cells for 16-24 h before imaging experiments to allow expression of the translocation reporter constructs (see Note 14).

\subsection{Time-lapse imaging of sub-membrane cAMP dynamics}

1. Rinse the cells on the coverslip once with the experimental buffer, add $2 \mathrm{~mL}$ fresh buffer to the dish and incubate for $30 \mathrm{~min}$ in a $37{ }^{\circ} \mathrm{C}$ incubator to allow cells to adapt to basal conditions in the experimental buffer.

2. Place the coverslip with cells in a superfusion chamber on the temperaturecontrolled microscope stage, connect the chamber to a peristaltic pump and superfuse cells with experimental buffer at a rate of $0.3-1 \mathrm{~mL} / \mathrm{min}$. The speed should be such that the entire chamber volume is exchanged several times per minute. Make sure that the temperature is $37^{\circ} \mathrm{C}$ in the chamber (see Note 15 ).

4. Observe the transfected cells in the fluorescence microscope with appropriate settings for fluorescent protein excitation and emission and select a region of the coverslip with cells expressing moderate levels of the two cAMP biosensor components. (see Note 16). 
5. Project the image on the CCD or CMOS camera and adjust acquisition parameters, such as exposure time and signal gain to appropriate levels for each spectral channel. (see Note 17)

6. Move the microscope stage to find a region on the coverslip without cells. Acquire a background image for each channel without changing imaging parameters. Return to the previously identified coverslip region with the cells.

7. Acquire image pairs with the CCD or CMOS camera every 1-5 s or other appropriate time interval.

8. Analyze changes of fluorescence in each of the two channels in individual cells. Most software permits plotting of intensity changes on-line, but more careful off-line analysis is usually required. Subtract the background images and generate CFP/YFP intensity ratio images (if the regulatory and catalytic components are labelled with CFP and YFP, respectively). Plot the intensity ratio from regions of interest using suitable software. Different analysis strategies are required depending on the type of images (see Note 18).

\subsection{Simultaneous imaging of sub-membrane $\mathrm{Ca}^{2+}$ and cAMP dynamics}

1. Rinse the transfected cells twice with experimental buffer, add $2 \mathrm{~mL}$ of the same buffer supplemented with $10 \mu \mathrm{M}$ of the acetoxymethyl ester of the $\mathrm{Ca}^{2+}$ indicator Fura Red and incubate for $45 \mathrm{~min}$ at $37^{\circ} \mathrm{C}$ and protect from light (see Note 19). 
2. Rinse the cells thoroughly with experimental buffer to remove extracellular indicator.

3. Mount the cover slip in the microscope superfusion chamber and record fluorescence as described in section 3.3 (see Fig. 4B and Notes 20 and 21).

\subsection{Simultaneous imaging of cAMP dynamics and subcellular localization of Epac2.}

1. Co-transfect the cells with the cAMP reporter components and a plasmid encoding mCherry-tagged Epac2 (24)(see section 3.2 above and Note 13). Use $1 \mu \mathrm{g}$ of mCherry-Epac2 plasmid DNA for each coverslip with cells to be transfected.

2. Image the cells as described in section 3.3 (see Note 22 and Fig 4C).

\section{Notes}

1. Instead of CFP, other fluorescent proteins can be used as long as the fluorescence from the regulatory and catalytic subunits can be reliably separated. Fluorescence from the regulatory subunit only serves as a reference and allows ratiometric recordings. However, if the experiment involves several fluorophores, the method also works without fluorescent labeling of the regulatory component. 
2. In case there is no fluorescent protein, (see Note 1) the membrane targeting polybasic region and CAAX-motif is added directly to the C-terminus of the PKA regulatory subunit.

3. The catalytic subunit always needs to be fluorescence-labeled since this is the part that undergoes translocation and therefore must be detected. Any fluorescent protein works well as long as it is spectrally distinct from that of the regulatory subunit. Note that the fluorescent proteins need not have overlapping emission and excitation spectra as for FRET sensors. The sensor does not need to be based on the catalytic C $\alpha$ subunit and the $C \beta$ works equally well.

4. This buffer is made freshly on the day of experiment.

5. Many suitable confocal systems are available through the major microscope manufacturers. The authors use a Yokogawa CSU-10 spinning disk system (available from several distributors in Europe and the US), well suited for live cell imaging in permitting a high acquisition speed and yet relatively low degree of photobleaching. TIRF illumination can be obtained through the objective lens using a high numerical aperture (NA) (>1.40) objective and beam focussing and positioning optics. Such TIRF illumination devices are available from most of the major microscope manufacturers. An alternative TIRF configuration uses a prism for illumination, which has the advantage of allowing imaging with a low magnification (10-20x) objective to obtain information from many cells in parallel (Fig. 3B). The authors use a custom-built system, but commercial illuminators are available from e.g. TIRF Labs Inc (Cary, NC). 
6. Commercial confocal microscopes are usually equipped with the necessary peripheral instrumentation but TIRF systems may require custom configuration.

7. A variety of diode-lasers, diode-pumped solid-state lasers and gas lasers suitable for exciting translocation reporters based on CFP/ YFP or GFP/mCherry are available from several companies. CFP is ideally excited with 445-nm diodes or with the 442 $\mathrm{nm}$ line of a helium-cadmium laser or even the $458 \mathrm{~nm}$ line of an argon laser. The $514.5 \mathrm{~nm}$ line of an argon laser or similar lines of solid-state lasers are the best for exciting YFP. GFP is excited with the 488-nm line of the argon laser or with solidstate lasers at e.g. 491 or $473 \mathrm{~nm}$. The red fluorescent protein mCherry is efficiently excited at $561 \mathrm{~nm}$ provided by many solid-state lasers.

8. Filters can be obtained from Chroma Technology (Bellows Falls, VT), Omega Optical (Brattleboro, VT) or Semrock (Rochester, NY). Dual channel recordings require a device for excitation wavelength switching, such as a filter wheel (e.g. from Sutter Instruments, Novato, CA) or an acousto-optic tunable filter (e.g. from AA Opto-electronic, Orsay, France). Dual wavelength emission recording requires either a filter wheel as above or an image splitter, such as the Photometrics Dual View DV-2 system (Tucson, Az).

9. Data acquisition and analysis software is usually included with commercial microscope systems but may have to be purchased separately for custom-built setups. The authors use MetaFluor from Molecular Devices (Sunnyvale, CA) and the free ImageJ software (26). 
10. It may be critical to maintain a temperature of $37^{\circ} \mathrm{C}$ during an experiment as well as to add and remove test substances. We use a peristaltic pump in combination with a custom-built superfusion chamber, chamber heater and microscope objective heater. Similar equipment is available from e.g. Warner Instruments (Hamden, CT).

11. As an alternative to poly-L-lysine, coverslips can be coated with collagen, fibronectin or laminin.

12. This protocol typically yields $30 \%$ transfection efficiency 1 day after transfection. The protocol is optimized for insulin-secreting MIN6 $\beta$-cells, and may have to be modified for other types of cells. For example, for some primary cells, including pancreatic $\beta$-cells, transduction with adenoviral vectors may be required.

13. All cDNA should be transfected at the same time to obtain maximal cotransfection. If more than two plasmids are used it is not recommended to use more than a total of $3 \mu \mathrm{g}$ plasmid DNA per coverslip as higher concentrations may have adverse effects and contribute to reduction of the transfection efficiency and interfere with cell function. Adjust the volume of liposomes when changing the amount of DNA to maintain the ratio of DNA:Lipofectamine 2000 at 1:2.5 (w/v). The plasmid DNA used for transfection must be of high quality. Our experience is that the quality of plasmid DNA correlates positively with the scale of production. We therefore recommend producing plasmid DNA from at least $200 \mathrm{~mL}$ bacterial cultures, corresponding to a standard "maxi-prep". 
14. Do not allow cells to express the biosensor protein for more than $24 \mathrm{~h}$, since excessive levels of the fusion proteins may affect cell function. This is because the cAMP-binding part of the reporter may buffer cAMP changes and the catalytic subunit remains catalytically active. We have tried to generate a kinase-dead mutant, but unfortunately the mutation somehow affected the ability of the protein to interact with the membrane-localized regulatory subunit.

15. Although cells can be stimulated by adding medium into the bath with a pipette, it is preferable to add medium using a superfusion system. Such a system not only permits convenient washout of the stimulus, but also eliminates problems with evaporation that otherwise would occur, since the ambient temperature in many cases should be maintained at $37{ }^{\circ} \mathrm{C}$ to observe normal physiological responses.

16. There will be cells expressing the fluorescent biosensors over a wide range of expression levels. High levels of cAMP-binding proteins and catalytically active PKA may potentially interfere with processes in the cells as mentioned in Note 14. Therefore choose cells with relatively low expression levels, approximately in the lower third of the brightness range of all cells. One should also consider that the two translocation reporter components are expressed at roughly equal levels. If there is a deficiency of the membrane-anchoring component, the catalytic subunit may bind exclusively to endogenous regulatory subunits at other subcellular locations. When these complexes dissociate following an elevation of the cAMP concentration, the signal at the plasma membrane may increase rather than decrease giving the impression of an inverted response. 
17. Select camera exposure time and gain settings so that no pixels in the image will be saturated. It is important to consider that the fluorescence intensity of the translocating PKA-C $\alpha$ component might change several-fold during an experiment. It is good to keep the exposure times as short as possible without compromising signalto-noise ratio, since excessive exposure to excitation light may result in photobleaching and phototoxic effects. If the fluorescence collected from the cells is very low, it is possible with most CCD cameras to combine charges in adjacent pixels to form one pixel in a process named binning. This will enhance the signal at the expense of optical resolution. On the contrary, if the signal is too bright, it indicates an excessive excitation light intensity. Reduce the laser power or attenuate the light with neutral density filters in the excitation beam path. The laser beam should be completely blocked with a shutter between image captures to avoid adverse effects of the light on the specimen.

18. a. For confocal microscopy images, plasma membrane translocation and dissociation of the biosensor can be quantified by placing a region of interest over the area corresponding to the plasma membrane, either manually or using a segmentation algorithm, such as a threshold or edge detection function. Measurements of membrane fluorescence may be difficult since the cell shape sometime changes over the time-course of an experiment, in particular after cell stimulation. An alternative is to quantify the changes in cytoplasmic fluorescence by defining a region of interest inside the cell that excludes the nucleus, membrane or other conspicuous organelles. This approach only works for analyzing changes in C $\alpha$-YFP fluorescence but not for the $\Delta$ RII $\beta$-CFPCAAX reference channel or for ratio images. 
b. In TIRF microscopy images, a region of interest over the cell will always show fluorescence in the plasma membrane and translocation or dissociation is simply recorded as changes of intensity or ratio in this region.

c. An increase of the sub-membrane cAMP concentration results in loss of C $\alpha$ YFP intensity but does not affect the $\triangle \mathrm{RII} \beta$-CFP-CAAX signal. The CFP/YFP ratio thus increases. If a ratio change is associated with changes in the $\Delta \mathrm{RII} \beta$ CFP-CAAX signal it indicates that there are also changes in cell adhesion or other unspecific effects of the stimulus.

d. The cAMP translocation biosensor is based on the dissociation of the catalytic and regulatory subunits of PKA, a process with a reported dissociation constant in the nanomolar to low micromolar range. This means that at very high cAMP concentrations, most of the subunits will have dissociated from each other and the sensitivity for detecting changes in cAMP will be reduced. This will not be a problem under most physiological conditions but if saturation is suspected, we recommend complementing the experiments with an alternative biosensor based on the low affinity cAMP-binding Epac proteins $(7,9,10)$.

e. To compensate for the differences in expression of the two reporter components, ratio changes can be expressed in relation to the baseline by dividing the ratio value at each time point with the prestimulatory level. In the case only the $\mathrm{C} \alpha$ reporter component is fluorescent the intensity changes can be expressed as fluorescence intensity changes in relation to baseline $\left(F / F_{0}\right.$ or $\left.F_{0} / F\right)$.

19. Dissolve Fura Red at $10 \mathrm{mM}$ in DMSO and store at $-20{ }^{\circ} \mathrm{C}$ protected from light. Mix before use and incubate together with cells at 2-20 $\mu \mathrm{M}$ during 20-45 minutes. The optimal indicator concentrations and incubation times vary depending on cell 
type and cell density. Excessive loading may reduce the $\mathrm{Ca}^{2+}$ response by buffering the concentration changes, whereas too little loading gives poor signal-to-noise ratio.

20. Fura Red is readily excited by the same light source as GFP or YFP, such as the 488- or 514.5-nm lines of the argon ion laser. Fluorescence emission is detected with a $630 \mathrm{~nm}$ long-pass filter. Upon $\mathrm{Ca}^{2+}$ binding, the excitation spectrum of Fura Red becomes blue-shifted resulting in loss of fluorescence excited at 488 and $514.5 \mathrm{~nm}$. Alternation between the Fura Red and cAMP translocation reporter emission filters using a filter wheel or similar device allows simultaneous measurements of cytoplasmic $\mathrm{Ca}^{2+}$ concentration and cAMP dynamics in the same cell. However, as YFP shows some emission even above $600 \mathrm{~nm}$, there may be a slight cross-over of YFP fluorescence into the Fura Red channel in cells with very bright YFP fluorescence and poor Fura Red loading. The degree of overlap is easily estimated by imaging cells expressing YFP, but lacking the $\mathrm{Ca}^{2+}$ indicator, using the Fura Red filter set. Alternatively, cAMP can be measured non-ratiometrically with an unlabelled regulatory subunit and a CFP-labelled C $\alpha$ subunit as illustrated in Fig 4B.

21. In recent years there has been substantial expansion of the genetically encoded $\mathrm{Ca}^{2+}$-indicator family, including the addition of two red-shifted versions that could be used together with the cAMP translocation biosensor. These two $\mathrm{Ca}^{2+}$ indicators, $\mathrm{R}$ GECO1 (27) and RCaMP (28) are readily excited by $561 \mathrm{~nm}$ excitation light while emission is measured $>620 \mathrm{~nm}$, providing complete separation from both CFP and YFP. The dynamic range, brightness and response amplitude of these indicators are significantly better than those of the $\mathrm{Ca}^{2+}$ dye Fura Red, but the genetically encoded indicators require the transfection of an additional plasmid. 
22. The mCherry fluorophore is excited by $561 \mathrm{~nm}$ laser light and emission is collected through a 584-nm long-pass filter (available from e.g. Semrock). Simultaneous recordings of mCherry, CFP and YFP fluorescence is difficult on most confocal or TIRF microscopy setups due to lack of suitable diochroic mirrors. Therefore, changes in cAMP will be non-ratiometrically recorded simply as changes in plasma membrane YFP fluorescence while the distribution of mCherry-Epac2 are recorded in parallel. Fluorescence from the CFP-tagged regulatory subunit anchored to the plasma membrane will not be recorded, precluding ratiometric measurements. Alternation between the mCherry and cAMP translocation reporter emission filters using a filter wheel or similar device allows simultaneous measurements of mCherry fluorescence and cAMP dynamics in the same cell.

\section{Acknowledgements}

We thank Drs Hongyan Shuai and Geng Tian for help with the preparation of Figure

4. The authors' work is supported by grants from the European Foundation for the Study of Diabetes, the family Ernfors Foundation, Novo Nordisk Foundation, the Swedish Diabetes Association and the Swedish Research Council (67X-14643, 67P21262, 325-2012-6778, 524-2013-298). 


\section{References}

1. Berridge M.J., Bootman, M.D., Roderick, H.L. (2003) Calcium signalling: dynamics, homeostasis and remodelling. Nat Rev Mol Cell Biol 4, 517-529

2. Willoughby D., Cooper, D.M. (2008) Live-cell imaging of cAMP dynamics. Nat Methods 5, 29-36

3. Berrera M., Dodoni, G., Monterisi, S. et al. (2008) A toolkit for real-time detection of cAMP: insights into compartmentalized signaling. Handb Exp Pharmacol 186, 285-298

4. Adams S.R., Harootunian, A.T., Buechler, Y.J. et al. (1991) Fluorescence ratio imaging of cyclic AMP in single cells. Nature 349, 694-697

5. Zaccolo M., De Giorgi, F., Cho, C.Y. et al. (2000) A genetically encoded, fluorescent indicator for cyclic AMP in living cells. Nat Cell Biol 2, 25-29

6. Zaccolo M., Pozzan, T. (2002) Discrete microdomains with high concentration of cAMP in stimulated rat neonatal cardiac myocytes. Science 295, 1711-1715

7. Nikolaev V.O., Bunemann, M., Hein, L. et al. (2004) Novel single chain cAMP sensors for receptor-induced signal propagation. J Biol Chem 279, 3721537218

8. Nikolaev V.O., Bunemann, M., Schmitteckert, E. et al. (2006) Cyclic AMP imaging in adult cardiac myocytes reveals far-reaching beta1-adrenergic but locally confined beta2-adrenergic receptor-mediated signaling. Circ Res 99, 1084-1091 9. Ponsioen B., Zhao, J., Riedl, J. et al. (2004) Detecting cAMP-induced Epac activation by fluorescence resonance energy transfer: Epac as a novel cAMP indicator. EMBO Rep 5, 1176-1180 
10. DiPilato L.M., Cheng, X., Zhang, J. (2004) Fluorescent indicators of cAMP and Epac activation reveal differential dynamics of cAMP signaling within discrete subcellular compartments. Proc Natl Acad Sci U S A 101, 16513-16518

11. Piston D.W., Kremers, G.J. (2007) Fluorescent protein FRET: the good, the bad and the ugly. Trends Biochem Sci 32, 407-414

12. Vogel S.S., Thaler, C., Koushik, S.V. (2006) Fanciful FRET. Sci STKE 2006, re2. doi 10.1126/stke.3312006re3312002

13. Rich T.C., Fagan, K.A., Nakata, H. et al. (2000) Cyclic nucleotide-gated channels colocalize with adenylyl cyclase in regions of restricted cAMP diffusion. $J$ Gen Physiol 116, 147-161

14. Dyachok O., Isakov, Y., Sågetorp, J. et al. (2006) Oscillations of cyclic AMP in hormone-stimulated insulin-secreting $\beta$-cells. Nature 439, 349-352

15. Scott J.D., Dessauer, C.W., Tasken, K. (2013) Creating order from chaos: cellular regulation by kinase anchoring. Annu Rev Pharmacol Toxicol 53, 187-210 16. Steyer J.A., Almers, W. (2001) A real-time view of life within $100 \mathrm{~nm}$ of the plasma membrane. Nat Rev Mol Cell Biol 2, 268-275

17. Dyachok O., Idevall-Hagren, O., Sågetorp, J. et al. (2008) Glucose-induced cyclic AMP oscillations regulate pulsatile insulin secretion. Cell Metab 8, 26-37 18. Tian G., Sandler, S., Gylfe, E. et al. (2011) Glucose- and hormone-induced cAMP oscillations in $\alpha$ - and $\beta$-cells within intact pancreatic islets. Diabetes 60, 1535 1543

19. Tian G., Sågetorp, J., Xu, Y. et al. (2012) Role of phosphodiesterases in the shaping of sub-plasma membrane cAMP oscillations and pulsatile insulin secretion. $J$ Cell Sci 125, 5084-5095 
20. Li J., Shuai, H.Y., Gylfe, E. et al. (2013) Oscillations of sub-membrane ATP in glucose-stimulated beta cells depend on negative feedback from $\mathrm{Ca}^{2+}$. Diabetologia 56, 1577-1586

21. Hansen C., Howlin, J., Tengholm, A. et al. (2009) Wnt-5a-induced phosphorylation of DARPP-32 inhibits breast cancer cell migration in a CREBdependent manner. J Biol Chem 284, 27533-27543

22. Malmersjö S., Liste, I., Dyachok, O. et al. (2010) $\mathrm{Ca}^{2+}$ and cAMP signaling in human embryonic stem cell-derived dopamine neurons. Stem Cells Dev 19, 13551364

23. Idevall-Hagren O., Barg, S., Gylfe, E. et al. (2010) cAMP mediators of pulsatile insulin secretion from glucose-stimulated single $\beta$-cells. J Biol Chem $\mathbf{2 8 5}$, $23007-23018$

24. Idevall Hagren O., Jakobsson, I., Xu, Y. et al. (2013) Spatial control of Epac2 activity by cAMP and $\mathrm{Ca}^{2+}$-mediated activation of Ras. Science Signaling 6, ra29. doi: $10.1126 /$ scisignal.2003932

25. Miyazaki J., Araki, K., Yamato, E. et al. (1990) Establishment of a pancreatic $\beta$ cell line that retains glucose-inducible insulin secretion: special reference to expression of glucose transporter isoforms. Endocrinology 127, 126-132 26. Schneider C.A., Rasband, W.S., Eliceiri, K.W. (2012) NIH Image to ImageJ: 25 years of image analysis. Nat Methods 9, 671-675

27. Zhao Y., Araki, S., Wu, J. et al. (2011) An expanded palette of genetically encoded $\mathrm{Ca}^{2+}$ indicators. Science 333, 1888-1891

28. Akerboom J., Carreras Calderon, N., Tian, L. et al. (2013) Genetically encoded calcium indicators for multi-color neural activity imaging and combination 
with optogenetics. Frontiers in molecular neuroscience 6, 2. doi:

$10.3389 /$ fnmol.2013.00002

\section{Figure legends}

Figure 1. Schematic illustration of a fluorescent translocation reporter for cAMP. (A) A truncated PKA RII $\beta$ regulatory subunit is fused to CFP with a polybasic sequence and a CAAX motif added to the $\mathrm{C}$-terminus to achieve membrane anchoring. The fullength PKA C $\alpha$ catalytic subunit is labelled with a C-terminal YFP. The amino acid sequences of the linker regions and the C-terminal membrane anchor are indicated. (B) Principle of operation of the translocation reporter. When the two moieties are co-expressed in a cell, C $\alpha$-YFP binds to the membrane-anchored $\Delta \mathrm{RII} \beta$ CFP-CAAX under basal conditions. When the cAMP concentration increases adjacent to the plasma membrane, $\mathrm{C} \alpha$-YFP dissociates from $\triangle \mathrm{RII} \beta$-CFP-CAAX and diffuses into the cytoplasm.

Figure 2. Confocal microscopy imaging of cAMP reporter protein translocation. (A) $\triangle \mathrm{RII} \beta$-CFP-CAAX (top row) and C $\alpha$-YFP (lower row) expressed in an insulinsecreting MIN6 $\beta$-cell, which is imaged before, during and after stimulation with 10 $\mathrm{nM}$ of the gluco-incretin hormone GLP-1. (B) Confocal images from a similar experiment with the phosphodiesterase inhibitor IBMX $(50 \mu \mathrm{M})$ as stimulus. The insets show magnifications of the areas outlined by the dashed rectangle. Note that $\Delta \mathrm{RII} \beta$-CFP-CAAX fluorescence remains constant at the membrane, whereas C $\alpha$-YFP translocates from the membrane to the cytoplasm in the presence of cAMP-elevating stimuli. Scale bars denote $10 \mu \mathrm{m}$. 
Figure 3. Recording of sub-membrane cAMP dynamics with TIRF microscopy. (A) $\triangle \mathrm{RII} \beta$-CFP-CAAX (top row), C $\alpha$-YFP (middle row) and the CFP/YFP ratio (bottom row) visualized in an insulin-secreting MIN6 $\beta$-cell before, during and after stimulation with $10 \mathrm{nM}$ GLP-1. The ratio images have been pseudo-coloured. Scale bar is $10 \mu \mathrm{m}$. (B) Low-magnification TIRF images of $\triangle \mathrm{RII} \beta-\mathrm{CFP}-\mathrm{CAAX}$ and $\mathrm{C} \alpha-$ YFP expressed in MIN6 $\beta$-cells as well as examples of single-cell time courses of CFP/YFP ratio changes from the same experiment. The cells are initially exposed to medium containing $3 \mathrm{mM}$ glucose and $250 \mu \mathrm{M}$ of the ATP-sensitive $\mathrm{K}^{+}$channel opener diazoxide. Depolarization by increasing the $\mathrm{K}^{+}$concentration to $30 \mathrm{mM}$ induces cAMP elevation in most cells, which mainly reflects $\mathrm{Ca}^{2+}$-stimulated cAMP production. Stimulation of cell metabolism by increase of the glucose concentration to $20 \mathrm{mM}$ markedly amplifies the cAMP responses. Scale bar denotes $100 \mu \mathrm{m}$.

Figure 4. Translocation reporter measurements of sub-membrane cAMP oscillations in $\beta$-cells stimulated with glucagon or glucose. (A) Ratiometric cAMP recording with the translocation sensor expressed in a primary $\beta$-cell at the surface of an intact mouse pancreatic islet. Addition of $10 \mathrm{nM}$ glucagon in the presence of $3 \mathrm{mM}$ glucose evokes pronounced slow oscillations of cAMP. The cAMP response is suppressed by $5 \mu \mathrm{M}$ adrenaline, which in $\beta$-cells acts mainly via $\alpha_{2}$-adrenergic receptors. The cAMP level is expressed as the ratio of $\triangle \mathrm{RII} \beta$-CFP-CAAX and $\mathrm{C} \alpha$-YFP fluorescence normalized to the intensity ratio under basal conditions. (B) Simultaneous recordings of cAMP with the translocation reporter and cytoplasmic $\mathrm{Ca}^{2+}$ concentration with the fluorescent dye Fura Red in an islet $\beta$-cell stimulated by an increase of the glucose concentration from 3 to $20 \mathrm{mM}$. To avoid spectral overlap between the fluorophores 
cAMP is recorded with a non-fluorescent membrane-anchoring component $(\Delta \mathrm{RII} \beta$ CAAX) and a CFP-labelled translocating component (C $\alpha$-CFP). The translocation recording is therefore non-ratiometric. Fluorescence intensities are expressed in relation to baseline $\left(\mathrm{F}_{0} / \mathrm{F}\right)$, with the scales inverted to illustrate $\mathrm{cAMP}$ and $\mathrm{Ca}^{2+}$ increases as positive deflections. (C) Simultaneous recordings of cAMP and plasma membrane binding of the cAMP effector protein Epac2 in a MIN6 $\beta$-cell stimulated by an increase of the glucose concentration from 3 to $11 \mathrm{mM}$. The cAMP recordings are performed non-ratiometrically as described in (B) but with YFP- instead of CFPlabelled $\mathrm{C} \alpha$. Epac2 was N-terminally tagged with mCherry and membrane fluorescence expressed in relation to baseline $\left(\mathrm{F} / \mathrm{F}_{0}\right)$. Increased signals indicate elevation of the cAMP concentration and increased plasma membrane binding of mCherry-Epac2. 
Fig. 1

A
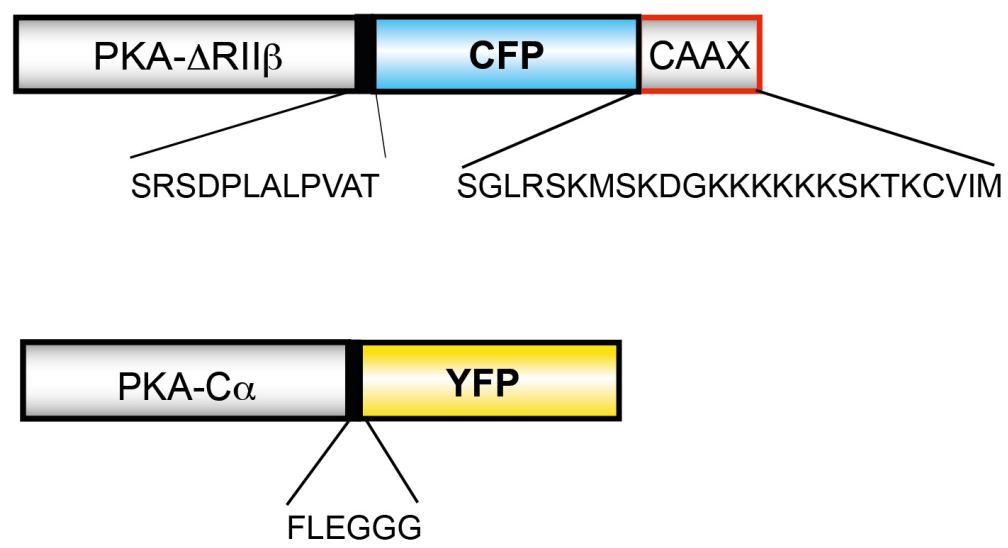

B

\section{$\mathbf{C}_{\alpha} \quad$ YFP}

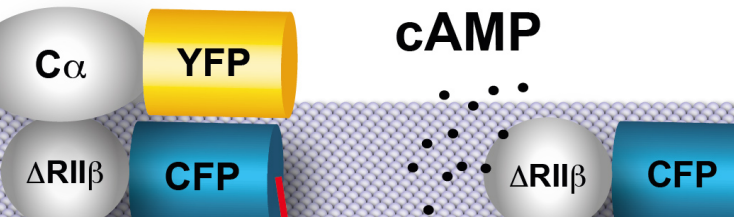


Fig. 2

A

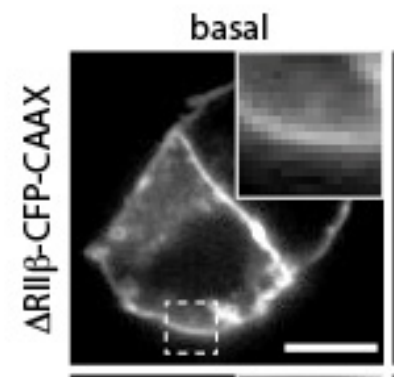

10 nM GLP-1
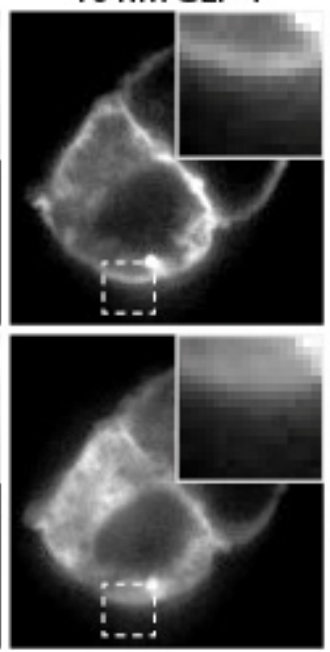

B
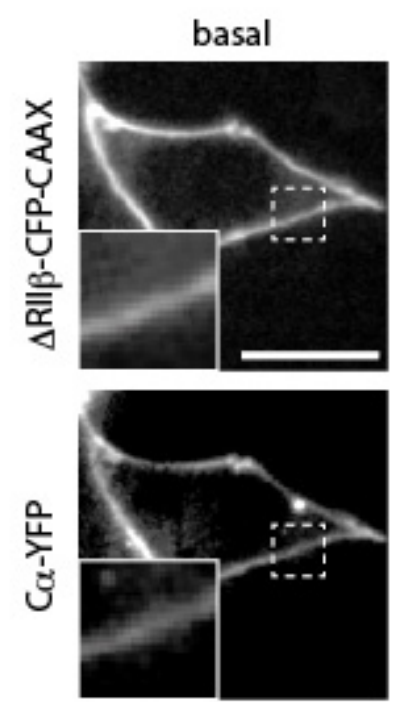

$50 \mu \mathrm{M}$ IBMX
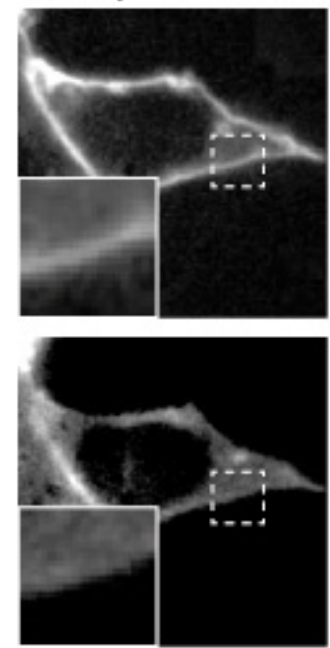

washout
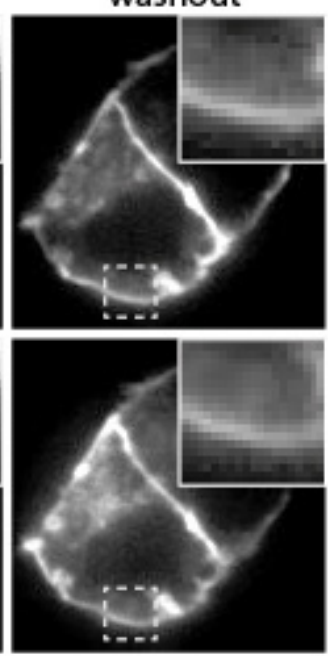

washout
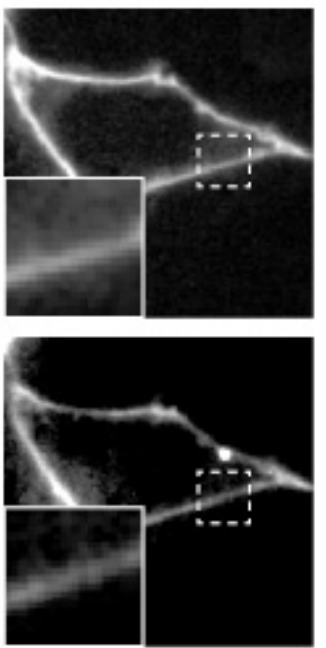
Fig. 3

A
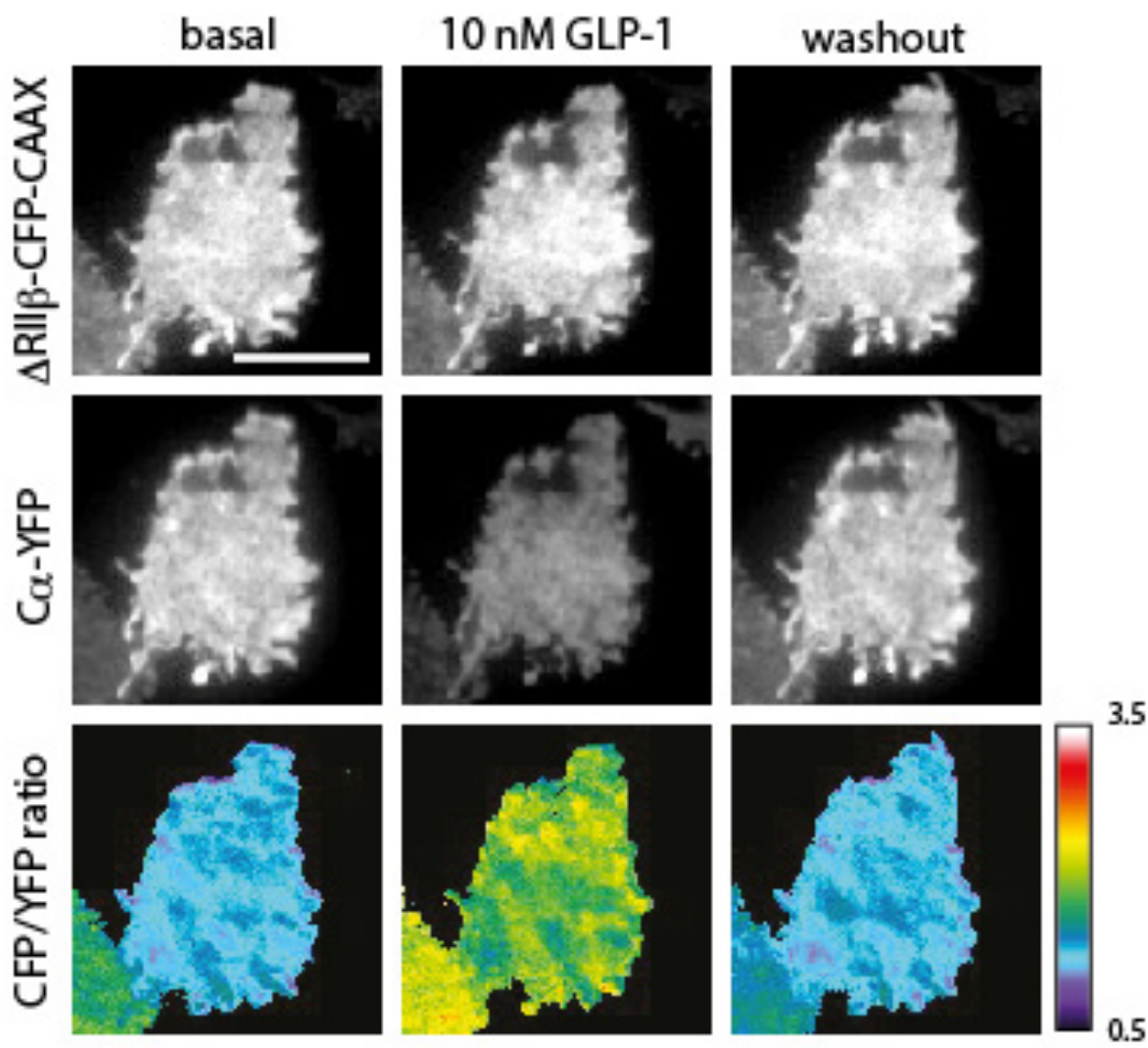

B

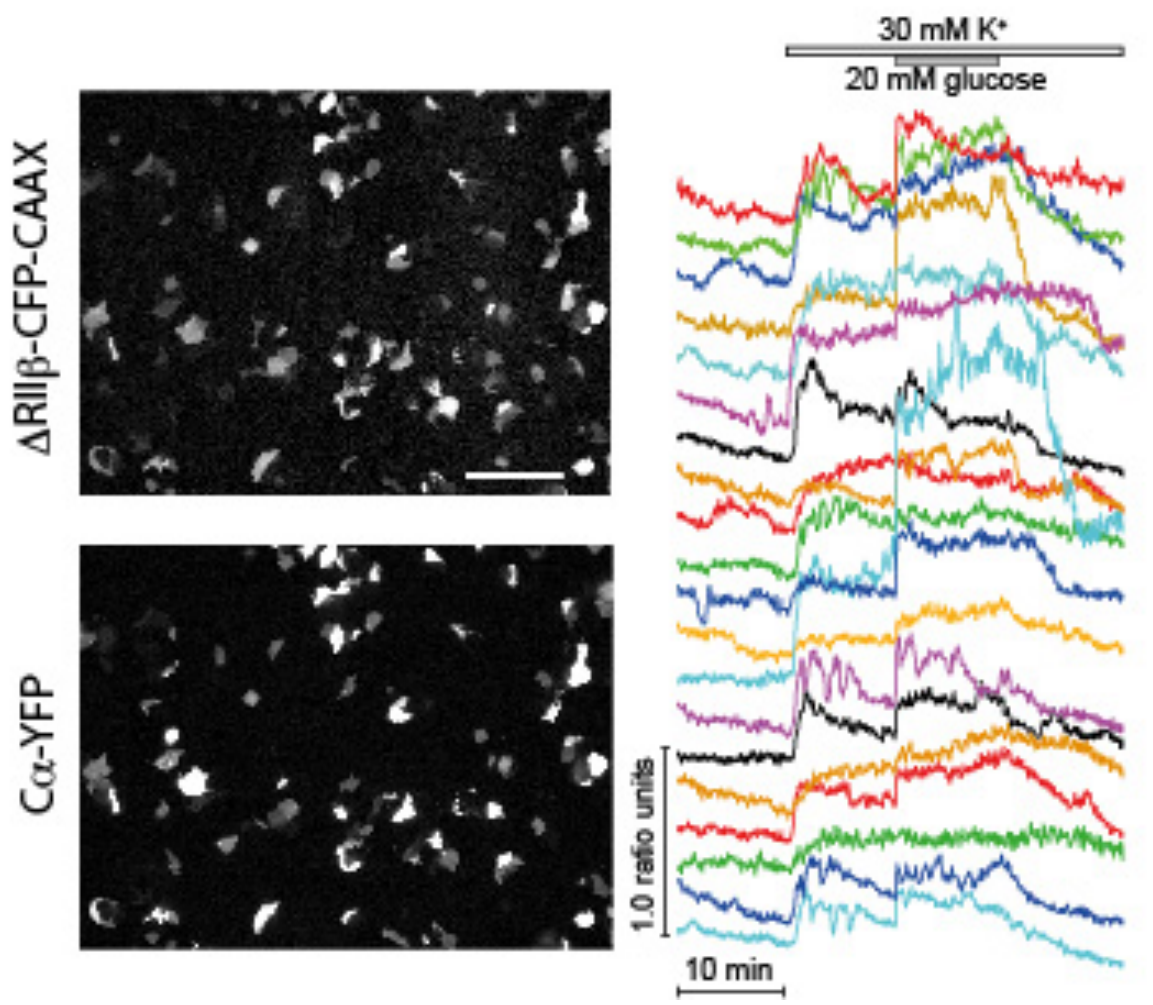


Fig. 4

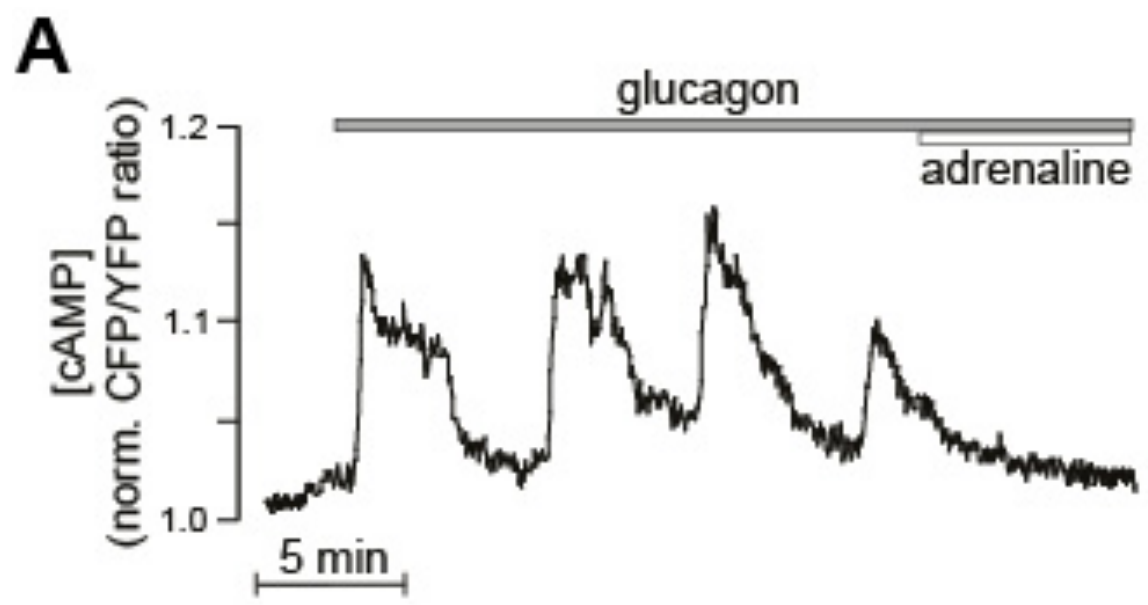

B

$20 \mathrm{mM}$ glucose
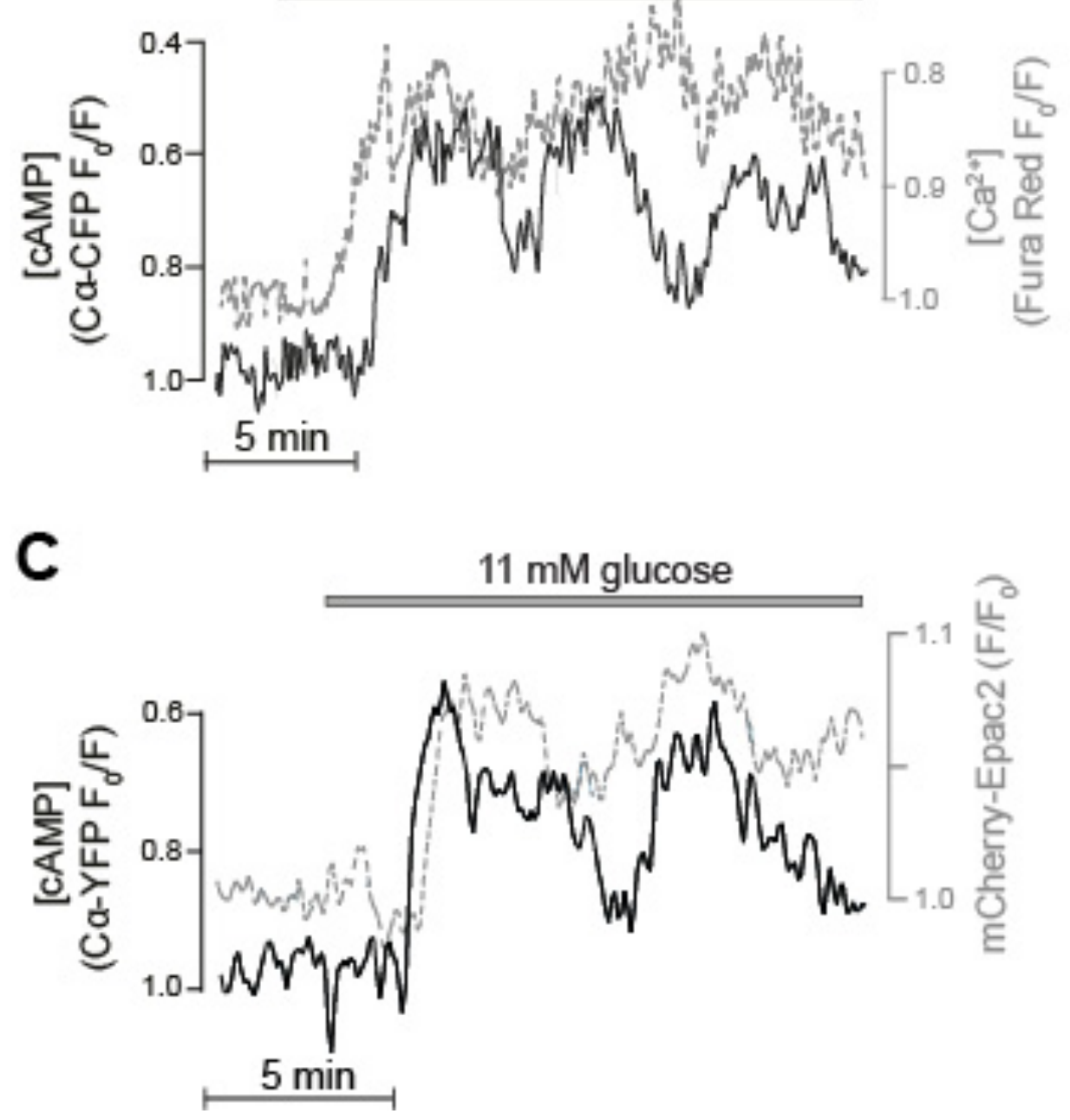